\title{
Microscale Fragmentation and Small-Angle Scattering from Mass Fractals
}

\author{
E. M. Anitas ${ }^{1,2}$ \\ ${ }^{1}$ Joint Institute for Nuclear Research, Dubna 141980, Russia \\ ${ }^{2}$ Horia Hulubei National Institute of Physics and Nuclear Engineering, 077125 Bucharest-Magurele, Romania \\ Correspondence should be addressed to E. M. Anitas; eanitasro@yahoo.com
}

Received 21 April 2015; Revised 29 July 2015; Accepted 30 July 2015

Academic Editor: Fajun Zhang

Copyright ( 2015 E. M. Anitas. This is an open access article distributed under the Creative Commons Attribution License, which permits unrestricted use, distribution, and reproduction in any medium, provided the original work is properly cited.

\begin{abstract}
Using the small-angle scattering method, we calculate here the mono- and polydisperse structure factor from an idealized fragmentation model based on the concept of renormalization. The system consists of a large number of fractal microobjects which are randomly oriented and whose positions are uncorrelated. It is shown that, in the fractal region, the monodisperse form factor is characterized by a generalized power-law decay (i.e., a succession of maxima and minima superimposed on a simple power-law decay) and whose scattering exponent coincides with the fractal dimension of the scatterer. The present analysis of the scattering structure factor allows us to obtain the number of fragments resulted at a given iteration. The results could be used to obtain additional structural information about systems obtained through microscale fragmentation processes.
\end{abstract}

\section{Introduction}

The number of fragments (such as those resulted from rocks weathering and explosions or produced by earth's crust) as a function of their sizes over a wide range of scales usually can be described by a fractal distribution [1-3], which is responsible for various physical properties, such as hydraulic conductivity or moisture characteristics in soils [4]. Although a quantification of these processes using the renormalization group approach has been suggested in $[5,6]$, an important issue concerns the distribution of fragments at microscales obtained by various methods such us in ultrasonic fragmentation using optoacoustic lens [7].

Small-angle scattering (SAS; neutrons, X-ray, light) $[8,9]$ is one of the most important techniques for investigating the microstructure of various types of systems which addresses the issue of size distribution, including the smallest and largest components. It yields the differential elastic cross section per unit solid angle as a function of the momentum transfer. The main advantage consists in its ability to differentiate between mass and surface fractals $[10,11]$, and it has been successfully used in studying the property of self-similarity across nano- and microscales [2], such as various types of membranes [12-15], cements [16], semiconductors [17], magnetic structures $[18,19]$, or biological structures [20-22]. Thus the concept of fractal geometry coupled with SAS technique can give new insights concerning the structural characteristics of such complex systems $[10,11,23-30]$. One of the main parameters which can be obtained is the fractal dimension $D_{m}$ [1]. For a mass fractal it is given by the scattering exponent of the power-law SAS intensity $I(q) \propto q^{-D_{m}}$ where $0<D_{m}<3$. For deterministic fractals, additional information can be obtained such as scaling factor (from the period of $I(q) q^{D_{m}}$ in the logarithmic scale), the number of fractal iteration $m$ (which equals the number of periods of function $\left.I(q) \propto q^{-D_{m}}\right)$, and the total number $N_{m}$ of structural units of which the fractal is composed.

In this paper, we develop a theoretical model based on renormalization group approach which could describe various microscale fragmentation processes. We calculate analytically the mono- and polydisperse structure factor and show how to obtain the main structural parameter such as the fractal dimension and the number of fragments at a given iteration and show how to estimate the smallest and largest radius of the fractal from SAS data. 


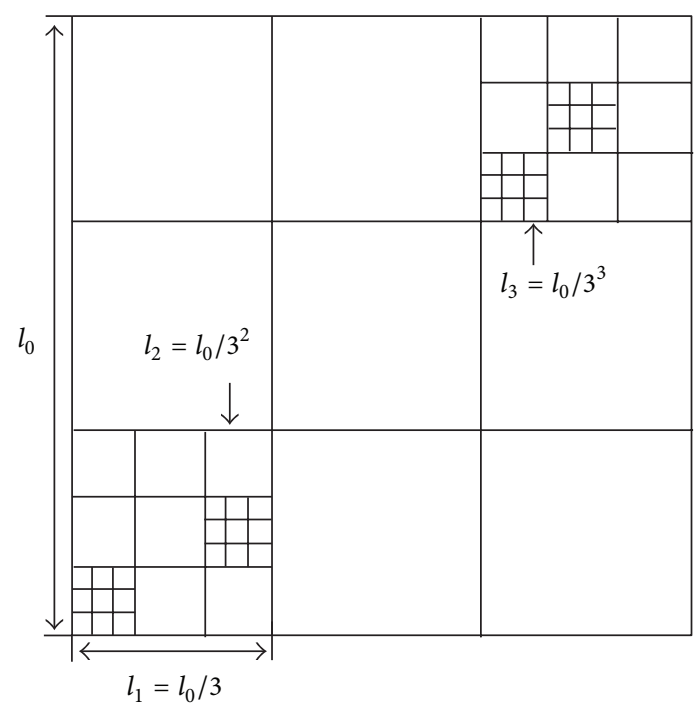

FIgURE 1: A two-dimensional projection of the fragmentation model. Note that at a given iteration all the fragments have the same size. The probability that the cube with edge length $l_{0}(m=0$; initiator) will be fragmented into 27 cubes of edge length $l_{1}$ is $f$.

\section{Fragmentation Model: Construction and Properties}

The construction process of three-dimensional mass fractals is similar to that of mass generalized Cantor and Vicsek fractals (GCF and GVF) [26] in the sense that one follows a top-down approach in which an initial structure is repeatedly divided (by a single scaling factor) into a set of smaller structures of the same type according to a given rule which is changed randomly from one iteration to the next one. Thus, we first consider a cube of edge length $l_{0}(m=0$; initiator $)$ and in the first iteration $(m=1)$ the number of cubes which are kept is related to the probability $f$ in which the initiator will be fragmented into 27 cubes of edge length $l_{1}=l_{0} / 3$. Figure 1 illustrates the construction of a generic model of fragmentation, where the sizes of remaining cubes at $m$ th iteration are given by

$$
l_{m}=\frac{l_{0}}{3^{m}}
$$

At $m$ th iteration the total number of particles will be given by

$$
N_{m}=(1-f)\left(1+27 f+(27 f)^{2}+\cdots+(27 f)^{m}\right) \text {, }
$$

and in a good approximation $(m \gg 1$ and $27 f>1)$ it can be shown that the fractal dimension can be written as [5]

$$
D_{m}=3 \frac{\log (27 f)}{\log 27}
$$

with $1 / 27<f<1$, and thus $0<D_{m}<3$. Figure 2 shows the variation of the fractal dimension $D_{m}$ with probability $f$ in which the initiator will be fragmented into 27 cubes of edge length $l_{1}=l_{0} / 3$.

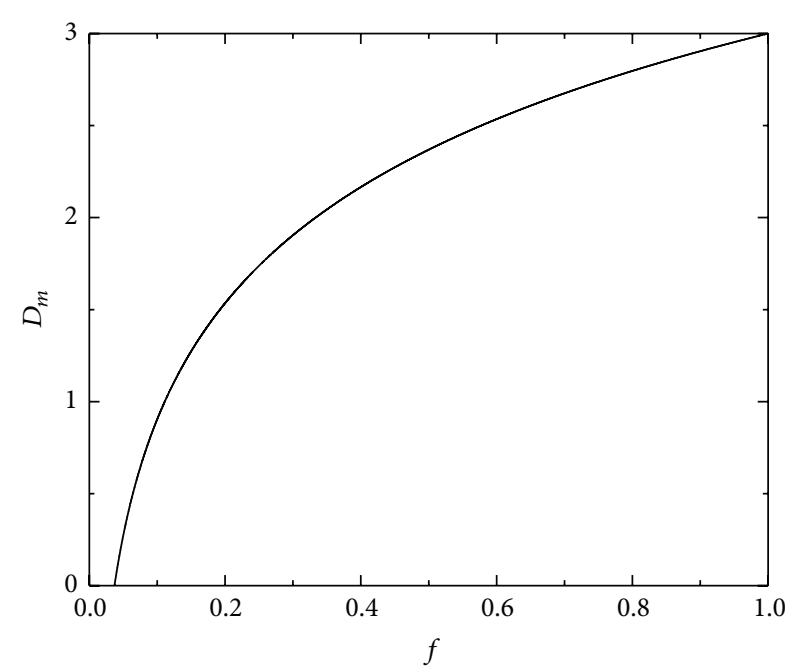

Figure 2: Dependence of the fractal dimension $D_{m}$ of the probability $f$ from (3).

\section{Theory}

Here, we restrict ourselves to two-phase systems, which are composed of homogeneous units of mass density $\rho_{m}$. The units are immersed into a solid matrix of pore density $\rho_{p}$. Then $[8,9]$ we can consider the system as if the units were frozen in a vacuum and had the density $\Delta \rho=\rho_{m}-\rho_{p}$. The density $\Delta \rho$ is called scattering contrast, and thus the scattering intensity is given by

$$
I(q)=n|\Delta \rho|^{2} V^{2}\left\langle|F(\mathbf{q})|^{2}\right\rangle,
$$

where $n$ is the fractal concentration, $V$ is the volume of each fractal, and $F(\mathbf{q})$ is the normalized form factor

$$
F(\mathbf{q})=\left(\frac{1}{V}\right) \int_{V} e^{-i \mathbf{q} \cdot \mathbf{r}} \mathrm{d} \mathbf{r},
$$

obeying $F(0)=1$. The brackets $\langle\cdots\rangle$ stand for the ensemble averaging over all orientations of $\mathbf{q}$. Once a deterministic fractal is composed of the same objects, say, $N$ cubes of edge length $l$, then

$$
F(\mathbf{q})=\frac{\rho_{\mathbf{q}} F_{0}(q l)}{N}
$$

with $\rho_{\mathbf{q}}=\sum_{j} e^{-i \mathbf{q} \cdot \mathbf{r}_{j}}$ being the Fourier component of the density of the cubes, and $\mathbf{r}_{j}$ are the center-of-mass positions of cubes. Here, the cube form factor of unit edge length is given by [8]

$$
F_{0}(\mathbf{t})=\frac{\sin \left(t_{x} / 2\right)}{t_{x} / 2} \frac{\sin \left(t_{y} / 2\right)}{t_{y} / 2} \frac{\sin \left(t_{z} / 2\right)}{t_{z} / 2}
$$

Therefore, the scattering intensity becomes

$$
I(q)=\frac{I(0) S(q)\left|F_{0}(q l)\right|^{2}}{N}
$$




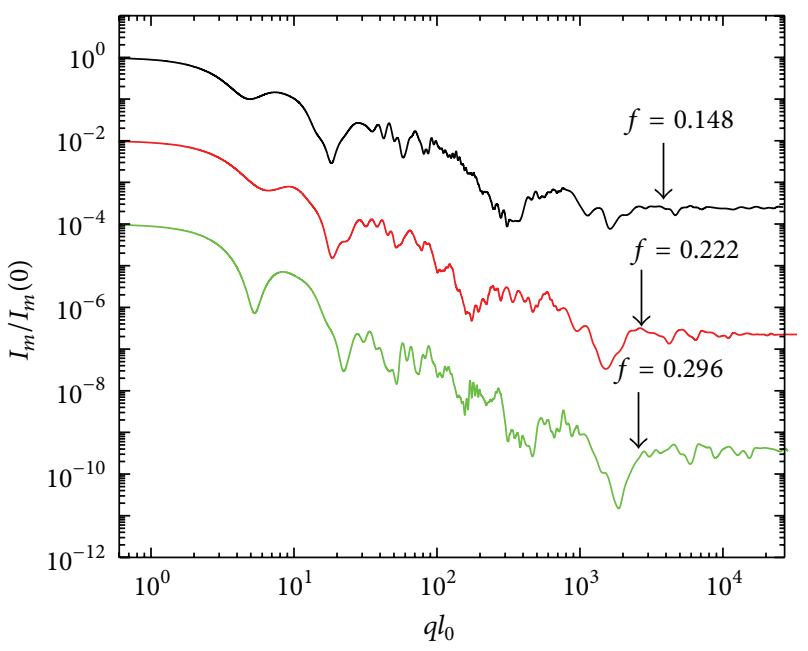

Figure 3: The monodisperse structure factor for the fragmentation model at various values of probability $f$ shows a superposition of maxima and minima on a simple power-law decay in the fractal region. The curves are shifted down vertically for clarity with a factor of $10^{-2}$ (red; middle curve) and, respectively, with $10^{-4}$ (green; lower curve). Iteration number is $m=6$.

with $I(0)=n|\Delta \rho|^{2} V^{2}$ and

$$
S(q) \equiv \frac{\left\langle\rho_{\mathbf{q}} \rho_{-\mathbf{q}}\right\rangle}{N}
$$

being the structure factor, and it is intimately connected to the pair distribution function. In the fractal region the form factor tends to one, and this implies that the scattering intensity is proportional to the structure factor in the fractal region.

The monodisperse form factor at $m$ th iteration can be written as [26]

$$
F_{m}(\mathbf{q})=F_{0}\left(q l_{m}\right) \prod_{i=1}^{m} G_{i}(\mathbf{q})
$$

with $m=1,2, \ldots$ and $G(\mathbf{q})$ being the generative function of the fractal and it specifies the positions of the scattering cubes inside the fractal. Thus, the scattering intensity becomes [26]

$$
\frac{I_{m}(q)}{I_{m}(0)}=\left\langle\left|F_{m}(\mathbf{q})\right|^{2}\right\rangle
$$

\section{Results and Discussion}

For well-known systems such as Cantor sets or Vicsek fractals $G_{i}(\mathbf{q})$ has known analytical expressions [26]. Here, we use a random distribution of cubes at each iteration for writing the generative function.

The monodisperse structure factor for various values of $f$ is shown in Figure 3. It is characterized by the presence of three main regions on a double logarithmic scale: at low $q$ $\left(q \lesssim 1 / l_{0}\right)$ a plateau (Guinier region) which gives information about the overall size of the fractal, a fractal (generalized

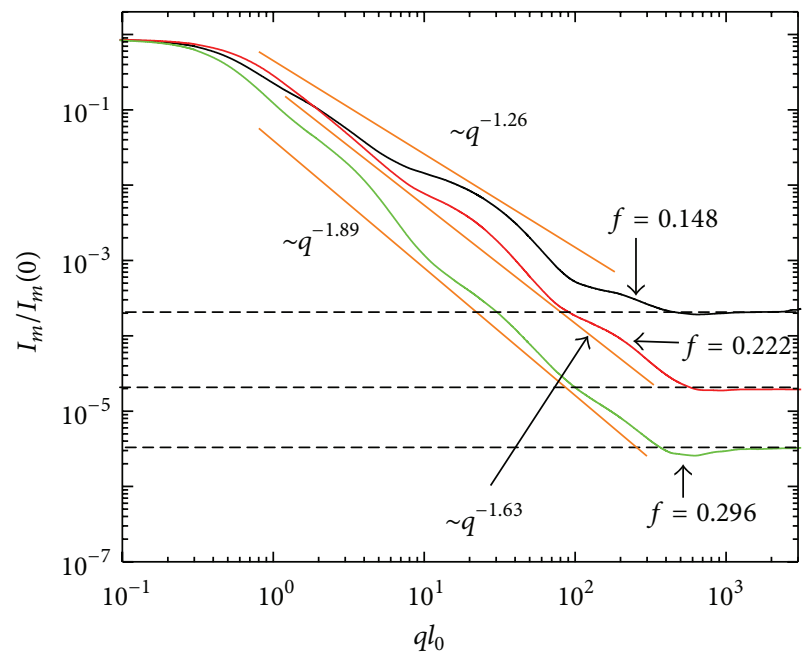

FIGURE 4: The polydisperse structure factor for the fragmentation model at various values of probability $f$ shows a simple power-law decay in the fractal region with the scattering exponent equal to the fractal dimension of the fractal. The horizontal lines indicate the asymptotic values of the structure factor (see (12)). Iteration number is $m=6$ and relative variance $\sigma_{r}=0.6$.

power law-decay) region at $1 / l_{0} \leqslant q \leqslant 1 / l_{m}$, and an asymptotic region

$$
S(q) \simeq \frac{1}{N_{m}}
$$

at high $q\left(1 / l_{m} \lesssim q\right)$ which gives information about the number of fragments in the fractal (see also Figure 4).

The value of the fractal dimension can be obtained by considering the polydispersity in size of the fractals. Here, we use a distribution function $D_{N}(l)$ of sizes in such a way that $D_{N}(l) \mathrm{d} l$ gives the probability of finding a fractal whose size falls within $(l, l+\mathrm{d} l)$. We consider a log-normal distribution

$$
D_{N}(l)=\frac{1}{\sigma l(2 \pi)^{1 / 2}} \exp \left(-\frac{\left[\ln \left(l / l_{0}+\sigma^{2} / 2\right)\right]^{2}}{2 \sigma^{2}}\right),
$$

where $\sigma=\left[\ln \left(1+\sigma_{r}^{2}\right)\right]^{1 / 2}$. The mean length and its relative variance are $\mu \equiv\langle l\rangle_{D}$ and $\sigma_{r} \equiv\left(\langle l\rangle_{D}^{2}-l_{0}^{2}\right)^{1 / 2} / \mu$ where $\langle\cdots\rangle_{D}=\int_{0}^{\infty} \cdots D_{N}(l) \mathrm{d} l$. Thus, the polydisperse structure factor is given by [26]

$$
I_{m}(q)=n|\Delta \rho|^{2} \int_{0}^{\infty}\left\langle\left|F_{m}(\mathbf{q})\right|^{2}\right\rangle V_{m}^{2} D_{N}(l) \mathrm{d} l,
$$

where $V_{m}$ is the total volume at the $m$ th iteration.

Figure 4 shows the polydisperse structure obtained from Figure 3 together with (14). As expected, the influence of polydispersity is to smooth the scattering curves, and to preserve the scattering exponent in the fractal region. At high $q$ the structure factor is proportional to $1 / N_{m}$, according to (12). Note that the value of fractal dimension is in agreement with (3). 
The results show that in an ideal SAS experiment (which shows the presence of Guinier, fractal, and Porod regions) the overall size and the smallest size in the fractal can be obtained from both the mono- and polydisperse intensity, through the position of the crossover between Guinier and fractal region, and, respectively, between fractal and Porod region. However, a reliable estimation of the fractal dimension can be obtained only in the polydisperse case.

An important difference between the model developed here and those based on exact self-similar fractals is that for the latter models we have a periodic distribution for the minima of monodisperse intensity [26, 29, 31], which allows us to obtain the number of fractal iteration, as well as the fractal scaling factor $\beta_{s}$ from the log-periodicity of the quantity $I(q) q^{D_{m}}$ versus $q$ in the fractal region. For the former model, the choice of the scattering units (here, cubes) according to a probability $f$ leads to an asymmetric distribution with respect to the origin of the initiator. This difference is reflected in the polydisperse intensity by oscillations of various amplitudes (see Figure 4) around the simple power-law decay $I(q) \propto$ $q^{-D_{m}}$. However, the asymptote of the fractal structure factor gives the number of particles $N_{m}$ inside the fractal and this can be used in (2), together with probability $f$ from (3) (with $D_{m}$ being known from the exponent of the power-law decay), to determine the iteration number $m$ of the fractal. Thus, by knowing $m, N_{m}$, and $D_{m}$ and using the relation [26] $N_{m}=$ $\left(1 / \beta_{s}\right)^{m D_{m}}$, we can then still recover the scaling factor.

Depending on the type and availability of the sample and on the experimental setup, various approaches could be followed in order to extract structural information. Thus, if the structure factor given by (12) is multiplied by a form factor corresponding to the basic scattering unit, then one obtains the SAS intensity in which the asymptotic region of the structure factor is replaced by a Porod decay $\left(I(q) \propto q^{-4}\right)$ and thus additional information can be obtained. For example, in [32], from the calculated small-angle X-ray scattering intensity of a fragmentation, fractal silica nanoagglomerates, the fractal structure, and aggregation number have been obtained by using the well-known Beaucage model [33], and the specific surface area has been obtained with the help of the integral parameter $Q$ and the Porod constant [9].

\section{Conclusions}

We have developed an idealized statistical self-similar fractal model for describing fragmentation processes based on 3D fractal and have used the SAS technique to characterize the microstructural properties of such fragments. The model is based on the concept of renormalization, and the suggested approach has important advantages since SAS is a nondestructive technique, the obtained information is averaged over a macroscopic volume, and the model features allow us to obtain additional information about the fractal fragments, such as the number of structural units of which the fractal is composed, the scaling factor or the fractal iteration number.

We have calculated the monodisperse and polydisperse structure factor and have shown how to obtain the smallest and largest sizes inside the fractal, from the position of the crossover between Guinier and fractal region, and, respectively, between fractal and Porod region. We have shown that the slope of SAS intensity is in agreement with the fractal dimension of the fractal, as given by (3).

The model can be used in understanding various physical properties such as hydraulic conductivity or moisture characteristics for systems obtained through fragmentation and it can be easily extended to describe more complex structures, such as by keeping fragments of different sizes obtained at various iterations (surface fractals), by taking into account multiple scaling factors at a given iteration (multifractals), changing the scaling factors with the iteration number according to a certain rule (fat fractals) or a combination of them.

\section{Conflict of Interests}

The author declares that there is no conflict of interests regarding the publication of this paper.

\section{Acknowledgment}

The author acknowledges financial support from JINR-IFIN$\mathrm{HH}$ projects awarded by the Romanian Plenipotentiary Representative at JINR.

\section{References}

[1] B. B. Mandelbrot, The Fractal Geometry of Nature, W.H. Freeman, 1983.

[2] J.-F. Gouyet, Physics and Fractal Structures, Springer, 1996.

[3] T. Vicsek, Fractal Growth Phenomena, World Scientific Publishing Co., Inc., Teaneck, NJ, Singapore, 1989.

[4] M. Bittelli, G. S. Campbell, and M. Flury, "Characterization of particle-size distribution in soils with a fragmentation model," Soil Science Society of America Journal, vol. 63, no. 4, pp. 782$788,1999$.

[5] D. L. Turcotte, "Fractals and fragmentation," Journal of Geophysical Research, vol. 91, no. 2, pp. 1921-1926, 1986.

[6] D. L. Turcotte, Fractals and Chaos in Geology and Geophysics, Cambridge University Press, 2nd edition, 1997.

[7] H. W. Baac, J. G. Ok, A. Maxwell et al., "Carbon-nanotube optoacoustic lens for focused ultrasound generation and highprecision targeted therapy," Scientific Reports, vol. 2, article 989, 2012.

[8] O. Glatter and O. Kratky, Small-Angle X-Ray Scattering, Academic Press, London, UK, 1982.

[9] L. A. Feigin and D. I. Svergun, Structure Analysis by Small-Angle $X$-Ray and Neutron Scattering, Springer, New York, NY, USA, 1987.

[10] J. E. Martin and A. J. Hurd, "Scattering from fractals," Journal of Applied Crystallography, vol. 20, no. 2, pp. 61-78, 1987.

[11] P. W. Schmidt, "Small-angle scattering studies of disordered, porous and fractal systems," Journal of Applied Crystallography, vol. 24, part 5, pp. 414-435, 1991.

[12] M. Balasoiu, E. M. Anitas, I. Bica et al., "SANS of interacting magnetic micro-sized Fe particles in a Stomaflex creme polymer matrix," Optoelectronics and Advanced Materials-Rapid Communications, vol. 2, no. 11, pp. 730-734, 2008. 
[13] E. M. Anitas, M. Balasoiu, I. Bica, V. A. Osipov, and A. I. Kuklin, "Small-angle neutron scattering analysis of the microstructure of stomaflex crème-ferrofluid based elastomers," Optoelectronics and Advanced Materials_Rapid Communications, vol. 3, no. 6, pp. 621-625, 2009.

[14] V. Maneeratana, J. D. Bass, T. Azaïs et al., "Fractal inorganic-organic interfaces in hybrid membranes for efficient proton transport," Advanced Functional Materials, vol. 23, no. 22, pp. 2872-2880, 2013.

[15] H. Amjad, Z. Khan, and V. V. Tarabara, "Fractal structure and permeability of membrane cake layers: effect of coagulationflocculation and settling as pretreatment steps," Separation and Purification Technology, vol. 143, pp. 40-51, 2015.

[16] A. Das, S. Mazumder, D. Sen et al., "Small-angle neutron scattering as a probe to decide the maximum limit of chemical waste immobilization in a cement matrix," Journal of Applied Crystallography, vol. 47, no. 1, pp. 421-429, 2014.

[17] K. Cho, P. Biswas, and P. Fraundorf, "Characterization of nanostructured pristine and Fe- and V-doped titania synthesized by atomization and bubbling," Journal of Industrial and Engineering Chemistry, vol. 20, no. 2, pp. 558-563, 2014.

[18] M.-L. Craus, A. K. Islamov, E. M. Anitas, N. Cornei, and D. Luca, "Microstructural, magnetic and transport properties of $\mathrm{La}_{0.5} \mathrm{Pr}_{0.2} \mathrm{~Pb}_{0.3-\mathrm{x}} \mathrm{Sr}_{\mathrm{x}} \mathrm{MnO}_{3}$ manganites," Journal of Alloys and Compounds, vol. 592, pp. 121-126, 2014.

[19] T. Naito, H. Yamamoto, K. Okuda et al., "Magnetic ordering of spin systems having fractal dimensions: experimental study," The European Physical Journal B, vol. 86, article 410, 2014.

[20] I. Yadav, S. Kumar, V. K. Aswal, and J. Kohlbrecher, "Smallangle neutron scattering study of differences in phase behavior of silica nanoparticles in the presence of lysozyme and bovine serum albumin proteins," Physical Review E, vol. 89, no. 3, Article ID 032304, 9 pages, 2014.

[21] R. Gebhardt, "Effect of filtration forces on the structure of casein micelles," Journal of Applied Crystallography, vol. 47, no. 1, pp. 29-34, 2014.

[22] J. Maji, S. M. Bhattacharjee, F. Seno, and A. Trovato, "Melting behavior and different bound states in three-stranded DNA models," Physical Review E, vol. 89, no. 1, Article ID 012121, 2014.

[23] H. D. Bale and P. W. Schmidt, "Small-angle X-ray-scattering investigation of submicroscopic porosity with fractal properties," Physical Review Letters, vol. 53, no. 6, pp. 596-599, 1984.

[24] P. W. Schmidt and X. Dacai, "Calculation of the small-angle Xray and neutron scattering from nonrandom (regular) fractals," Physical Review A, vol. 33, article 560, 1986.

[25] J. Teixeira, "Small-angle scattering by fractal systems," Journal of Applied Crystallography, vol. 21, no. 6, pp. 781-785, 1988.

[26] A. Y. Cherny, E. M. Anitas, V. A. Osipov, and A. I. Kuklin, "Deterministic fractals: extracting additional information from small-angle scattering data," Physical Review E, vol. 84, no. 3, Article ID 036203, 2011.

[27] D. Günther, D. Y. Borin, S. Günther, and S. Odenbach, "X-ray micro-tomographic characterization of field-structured magnetorheological elastomers," Smart Materials and Structures, vol. 21, no. 1, Article ID 015005, 2012.

[28] A. Y. Cherny, E. M. Anitas, V. A. Osipov, and A. I. Kuklin, "Small-angle scattering from multiphase fractals," Journal of Applied Crystallography, vol. 47, no. 1, pp. 198-206, 2014.

[29] E. M. Anitas, "Small-angle scattering from fat fractals," The European Physical Journal B, vol. 87, p. 139, 2014.
[30] I. Bica, E. M. Anitas, L. M. E. Averis, and M. Bunoiu, "Magnetodielectric effects in composite materials based on paraffin, carbonyl iron and graphene," Journal of Industrial and Engineering Chemistry, vol. 21, pp. 1323-1327, 2015.

[31] E. M. Anitas, "Scattering structure factor from fat fractals," Romanian Journal of Physics, vol. 60, no. 5-6, p. 647, 2015.

[32] R. Wengeler, F. Wolf, N. Dingenouts, and H. Nirschl, "Characterizing dispersion and fragmentation of fractal, pyrogenic silica nanoagglomerates by small-angle X-ray scattering," Langmuir, vol. 23, no. 8, pp. 4148-4154, 2007.

[33] G. Beaucage, "Approximations leading to a unified exponential/power-law approach to small-angle scattering," Journal of Applied Crystallography, vol. 28, no. 6, pp. 717-728, 1995. 

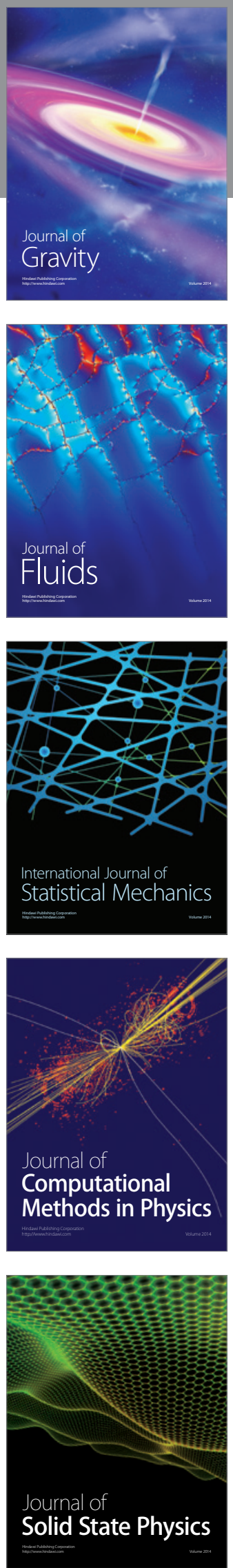

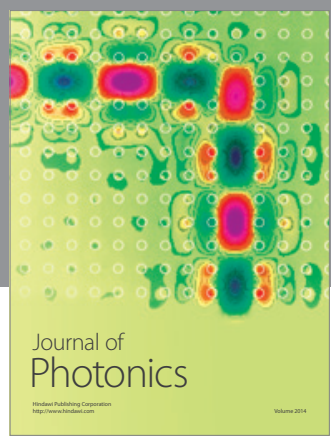

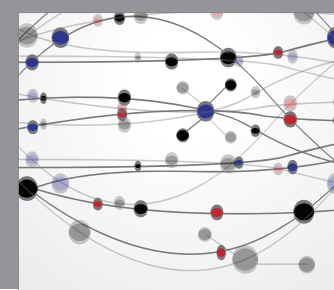

The Scientific World Journal

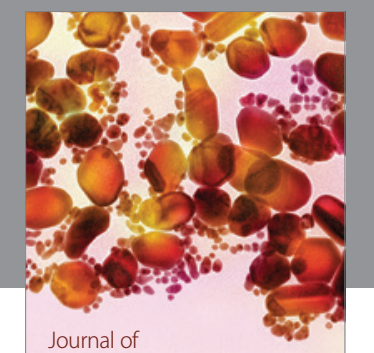

Soft Matter
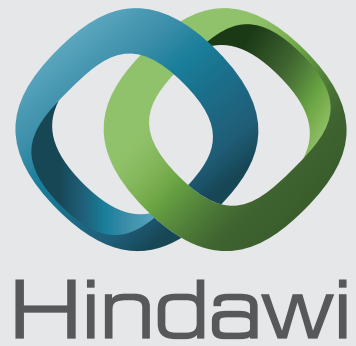

Submit your manuscripts at

http://www.hindawi.com
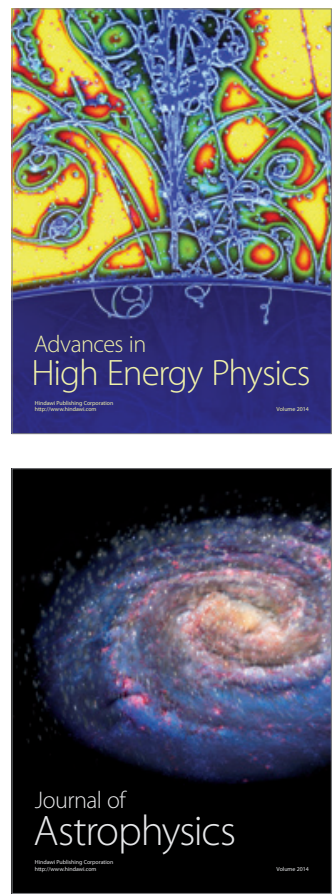
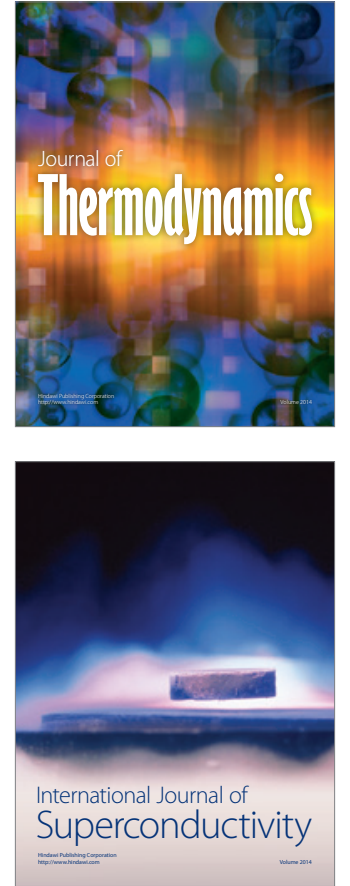
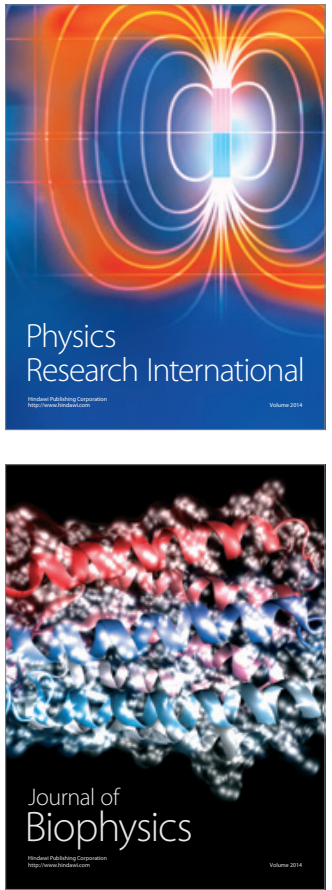
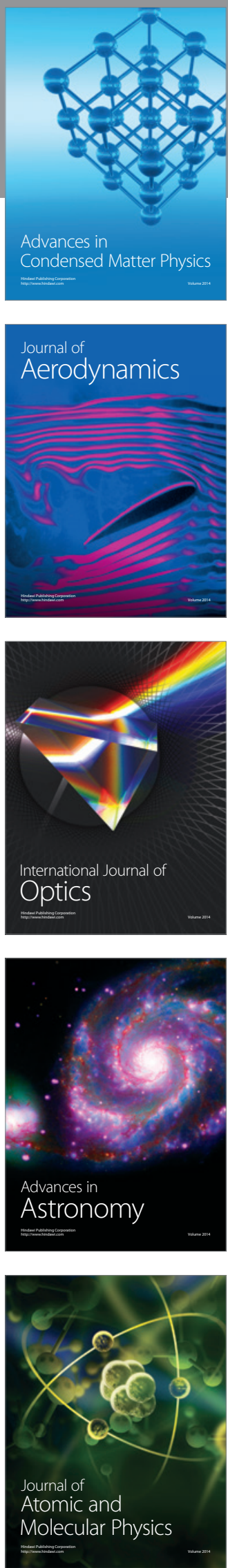